\title{
Leishmaniasis Mucocutánea con Manifestacion Oral: Reporte de un Caso
}

\author{
Mucocutaneous Leishmaniasis with Oral Manifestation: Case Report
}

Ignacio Javier Molina Avila'; Juan Martin Pimentel Sola'; María Fernanda García Bustos²; Julia Pimentel Sola; Diego Marco Jorge ${ }^{4}$; Guadalupe Brunetto ${ }^{5}$ Mauree Córdoba ${ }^{6} \&$ Karina Cordero Torres ${ }^{7}$

MOLINA, A. I. J.; PIMENTEL, S. J. M.; GARCÍA, B. M. F.; PIMENTEL, S. J.; MARCO, J. D.; BRUNETTO, G.; CÓRDOBA, M. \& CORDERO, T. K. Leishmaniasis mucocutánea con manifestacion oral: reporte de un caso. Int. J. Odontostomat., 13(3):342-347, 2020.

RESUMEN: La Leishmaniasis es un grupo de enfermedades transmitidas por vectores y causada por la Leishmania, un parásito intracelular, que se presenta de preferencia en regiones tropicales y subtropicales. Se manifiesta mediante un amplio rango de formas clínicas como la cutánea, mucocutánea, y visceral, dependiendo de la especie y respuesta inmunológica del paciente. Se presenta el caso de un hombre de 35 años que acudió derivado a Unidad de Estomatología del Hospital Señor del Milagro, Salta, Argentina, presentando en la cavidad oral lesión, granulomatosa, ulcerada, dolorosa a la palpación, única, en paladar blando, de tres meses de evolución. Se realizaron estudios serológicos, parasitológicos y PCR. Los ELISAs lisados, PCRs y cultivos de materiales de lesiones fueron positivos, confirmando diagnóstico de leishmaniasis mucocutánea. El paciente fue derivado al Servicio de Dermatología donde recibió tratamiento con Antimoniato de Meglumina, con repuesta clínica favorable. El conocimiento de las manifestaciones orales puede llevar al diagnóstico clínico de leishmaniasis mucocutánea por parte del odontólogo, pudiendo entregar un tratamiento oportuno y a la vez ayudar al paciente, evitando complicaciones de esta enfermedad.

PALABRAS CLAVE: leishmaniasis mucocutánea, leishmaniasis tegumentaria, espundia.

\section{INTRODUCCIÓN}

La leishmaniasis es una enfermedad parasitaria no contagiosa causada por protozoos flagelados del género Leishmania, trasmitida por la picadura de un insecto de la familia de Phlebotominae del género Lutzomyia en Argentina (ul Bari, 2006; Salomón et al., 2012; Sánchez-Romero et al., 2020). Esta enfermedad se clasifica como cutánea (LC), mucocutánea (LMC) y visceral (LV), con un amplio espectro de manifestaciones clínicas, relacionadas con: la especie infectante, el medio ambiente y respuesta inmunológica del huésped (Organización Mundial de la Salud, 2012). Strazulla y cols hablan del término leishmaniasis de mucosa (ML) y la describen como aquella forma de infección de los tejidos mucosos por
Leishmania spp. En particular, la ML implica membranas mucosas del tracto respiratorio superior (desde la pared nasal hacia la laringe) y la cavidad oral (Strazzulla et al., 2013).

Se ha estimado que, a nivel mundial, hay 12 millones de personas infectadas con Leishmania spp. y adicionalmente hay 350 millones con riesgo de infectarse (Lockard et al., 2019). En Argentina, se reportó que entre 2013 al 2017, se confirmaron 1295 casos, de los cuales $87,1 \%$ correspondían a LC. La LMC fue la segunda más frecuentes, con 115 casos reportados, principalmente den las regiones del norte y noreste de Argentina (Germano et al., 2019).

\footnotetext{
${ }^{1}$ Unidad de Estomatología, Hospital Señor del Milagro, Salta, Argentina.

2 Área Microbiología. Investigadora Asistente de CONICET, Instituto de Patología Experimental, Argentina.

${ }^{3}$ Becaria Doctoral de CONICET, Instituto de Patología Experimental, Argentina.

${ }^{4}$ Investigador Independiente de CONICET, Instituto de patología Experimental, Argentina.

${ }^{5}$ Hospital Señor del Milagro Salta, Argentina.

${ }^{6}$ Servicio de Dermatología, Hospital Señor del Milagro Salta Argentina

${ }^{7}$ Escuela de Ciencias de la Salud, Universidad Viña del Mar, Viña del Mar, Chile.
} 
La LMC generalmente ocurre después de la aparente resolución de la infección cutánea, aunque puede coexistir con afectación de la piel existiendo excepciones donde se manifiestan al mismo tiempo (Hoyos et al., 2019). Las lesiones mucosas normalmente se manifiestan dentro de los 2 años posteriores a la aparición cutánea, pero se describe incluso hasta 30 años posterior a la presentación cutánea (MontenegroIdrogo et al., 2014; Handler et al., 2015a).

La leishmaniasis afecta a hombres y mujeres en una proporción de 2:1. En pacientes inmunocomprometidos, la mucosa oral es el segundo sitio más frecuentemente afectado de la región de la cabeza y el cuello. En la cavidad oral, la lengua es el sitio más afectado. La LMC se debe a la extensión de la enfermedad local de la piel al tejido mucoso a través de la extensión directa, el torrente sanguíneo o los vasos linfáticos (Passi et al., 2014). Se caracteriza por lesiones granulomatosas, eritema y ulceras, presentándose en mucosa de nariz, cavidad oral y faringe, llevando a la destrucción del tabique nasal. En la cavidad oral, se manifiesta principalmente en paladar duro (Cruz de Escomel) pudiendo afectar labio, úvula, en-

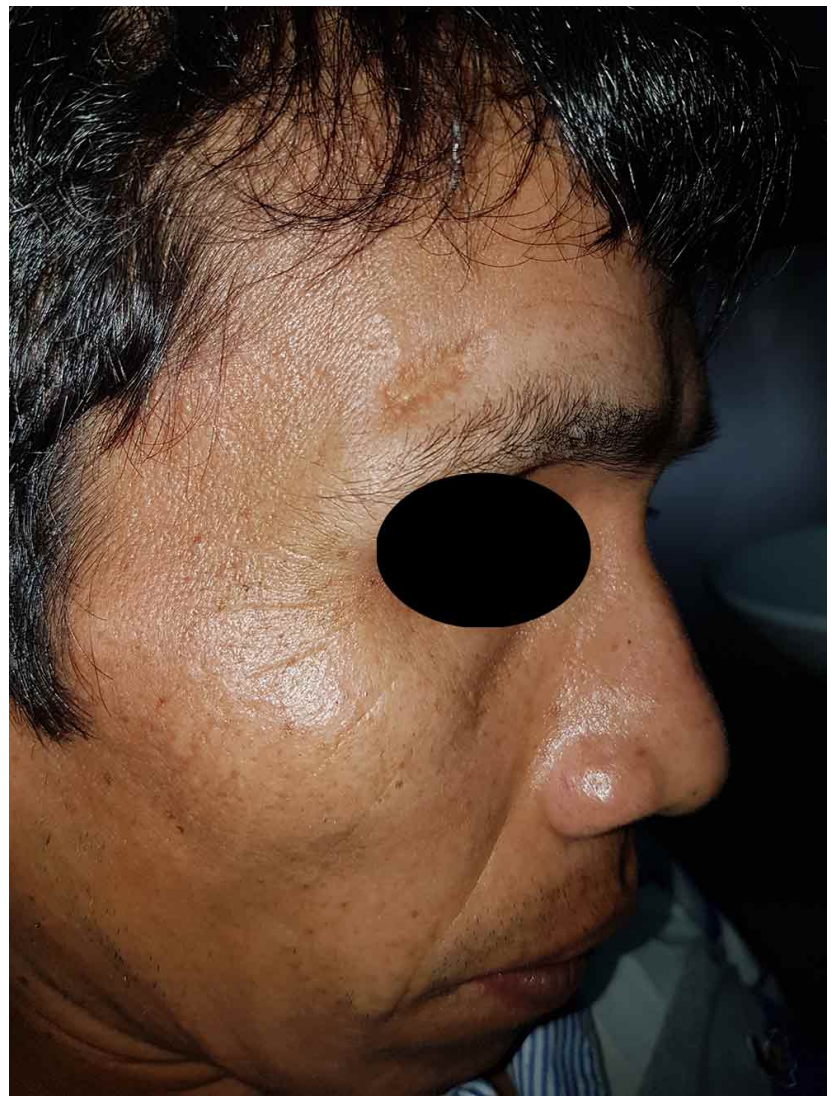

Fig. 1. Lesión cicatrizada de aspecto de "papel de cigarro" en la región supra orbitaria derecha. cía, lengua, amígdala y la región retromola (Motta et al., 2007). La afección de la mucosa es poco frecuente y resulta de la diseminación hematógena o linfática de amastigotes desde la piel hasta la nariz y orofaringe. Las manifestaciones orales únicas suelen ser difícil de diagnóstico, ya que la cantidad de amastigotes en las lesiones es en general escasa (Almeida et al., 2016).

Las principales pruebas diagnósticas son los métodos directos tales como: frotis, cultivo, biopsia, y PCR, y métodos indirectos: como inmunofluorescencia indirecta (IFI), y ELISA, aumentando la eficacia con la combinación de ambos métodos (Marco et al., 2012; Handler et al., 2015b).

El tratamiento de elección es el antimoniato de meglumina (Glucantime $\AA$ ), una o dos inyecciones intramusculares diarias durante 21 a 35 días, pudiendo presentar efectos secundarios y reacciones graves como toxicidad cardíaca, alteraciones hepáticas, pancreáticas, o renales (García Bustos et al., 2014).

\section{CASO CLÍNICO}

Se presentó a la consulta un paciente masculino de 35 años, albañil, oriundo de la ciudad de San Ramón de la Nueva Orán, Salta, y con residencia actual en ciudad de Salta Capital. El mismo fue derivado a la Unidad de Estomatología del Hospital Señor del Milagro, Salta, Argentina, por presentar lesión granulomatosa en paladar, de tres meses de evolución, no sangrantes y afebril, sin referir antecedentes personales patológicos y con diagnostico presuntivo de micosis profunda.

Refirió como antecedente, haber vivido hasta los 15 años de edad en zona endémica para enfermedades tropicales, en el Departamento de Orán, Salta. Al examen clínico cutáneo presentó lesión cicatrizada de aspecto de "papel de cigarro" en la región supra orbitaria derecha de aproximadamente 20 años, refiriendo que fue indolora y auto resolutiva, ya que no se realizó el diagnóstico, ni recibió tratamiento para LC alguno (Fig. 1). Se observó perforación con erosión parcial del tabique nasal (Fig. 2). No manifestó poliadenopatías al momento de la consulta.

A la inspección clínica intraoral se observó una lesión única granulomatosa de coloración rosa páli- 


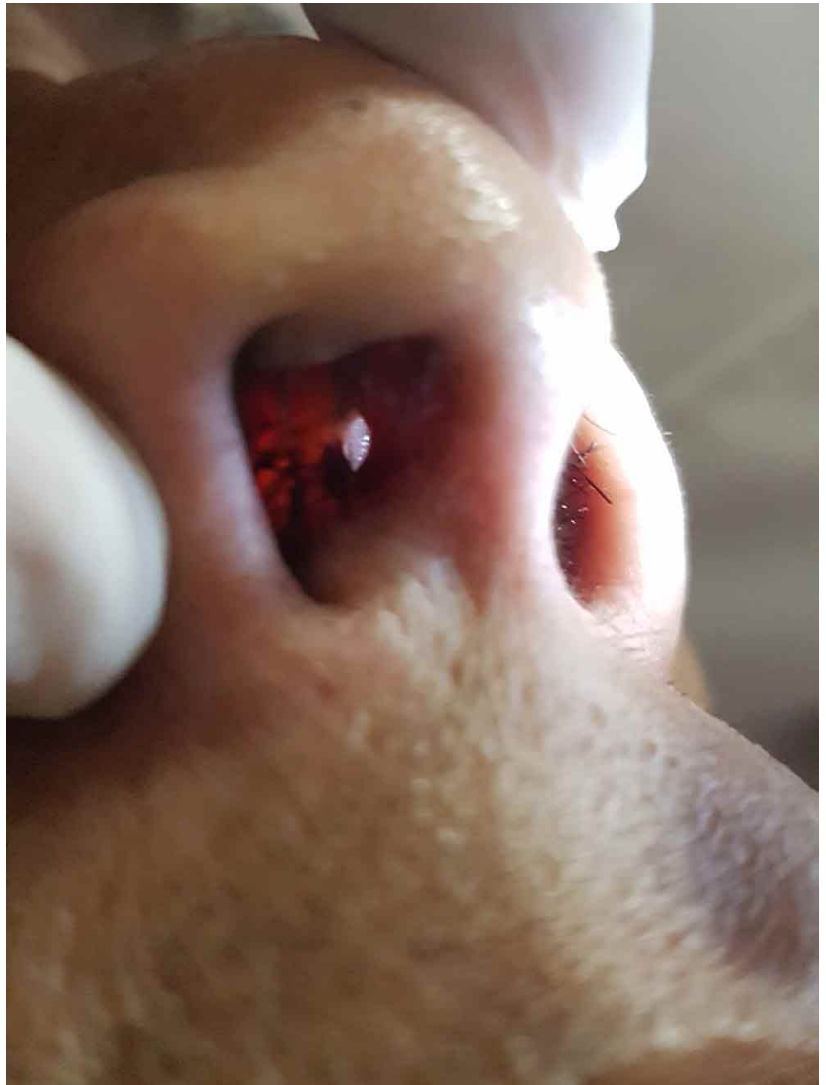

Fig.2. Perforación con erosión parcial del tabique nasal.

do con dolor a la palpación, no sangrante, que se extiendía desde el tercio posterior paladar del duro, hasta el paladar blando y región amigdalina, en forma de pirámide ("Cruz de Escomel”) (Fig. 3). No se palparon adenopatías cervicales. Se planteó LMC como diagnostico presuntivo.

Se realizó test de ELISA para VIH (Virus de Inmunodeficiencia Adquirida), dando como resultados no reactivos. Se descartó micosis profunda, siendo negativos los estudios: directos, biopsia, cultivos e inmunodifusión para paraccoccidioidomicosis e

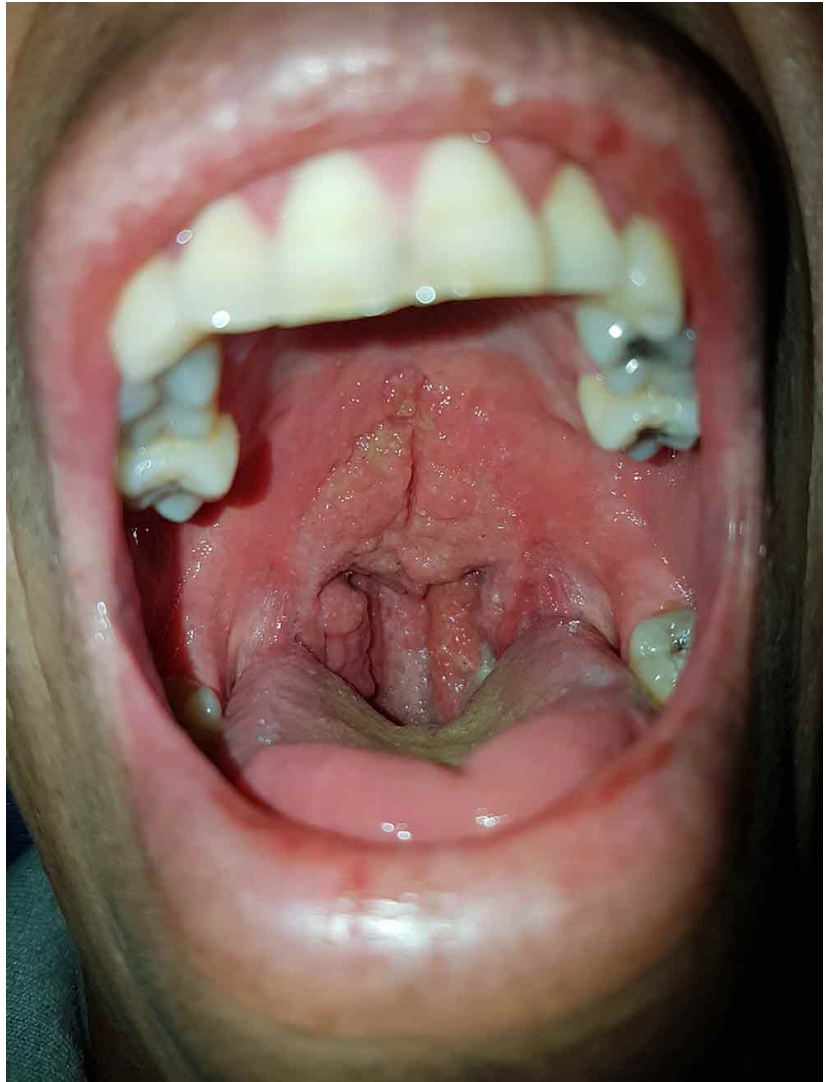

Fig. 3. Cruz de Escomel: Lesión granulomatosa dolorosa a la palpación, no sangrante.

histoplasmosis. Se realizó el estudio parasitológico para leishmaniasis en el Instituto de Patología Experimental de la Facultad de Ciencias de la Salud de la Universidad Nacional de Salta, con resultados negativos para frotis y cultivo, y positivos para ELISA y PCR para Leishmania spp (Fig. 4). Este patrón de resultados tiene un valor predictivo superior al $96 \%$ y es el más frecuente en el laboratorio de LMC (Tabla I). En una toma de muestra posterior, se logró aislar Leishmania spp. Se observó desarrollo de promastigotes en el medio bifásico USMARU-PBSS después de una semana de sembrado.

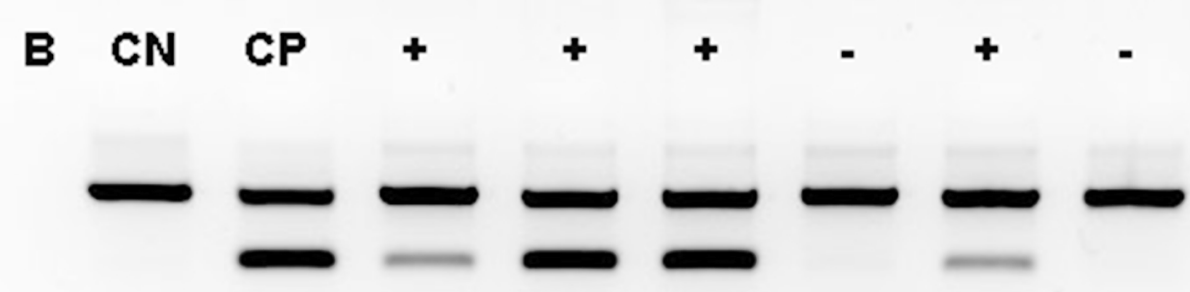

Fig. 4. Reacción en cadena de la Polimerasa (PCR). Gel de agarosa donde se evidencia amplificación (+) de fragmento de ADN kinetoplástico (kDNA) de Leishmania spp. $\mathrm{B}$ : blanco. CN: control negativo. CP: control positivo. (-): muestras negativas (sin amplificación). 
MOLINA, A. I. J.; PIMENTEL, S. J. M.; GARCíA, B. M. F.; PIMENTEL, S. J.; MARCO, J. D.; BRUNETTO, G.; CóRDOBA, M. \& CORDERO, T. K. Leishmaniasis mucocutánea con manifestacion oral: reporte de un caso. Int. J. Odontostomat., 13(3):342-347, 2020.

Tabla I. Valores predictivos para el estudio y diagnóstico diferencial de Paracoccidiodomicosis, Histoplasmosis y Leishmaniasis mucocutánea.

\begin{tabular}{llll}
\hline & Paracoccidioidomicosis & Histoplasmosis & Leishmaniasis Mucocutánea \\
\hline istopatología & $(-)$ & $(-)$ & $(-)$ \\
xamen Directo & $(-)$ Paracoccidioides spp & $(-)$ Histoplasma capsulatum & $(-)$ Mucosa oral Amastigote \\
ultivo & $(-)$ & $(-)$ & $(+)$ \\
rueba Serológica & $(-)$ inmunodifusión & $(-)$ inmunodifusión & $(+)$ Elisa (lisado de promastigotes de $L$. \\
& & & $($ V.) braziliensis \\
tros Estudios & & & $(+)$ PCR ORAL (leishmania spp.) \\
\hline
\end{tabular}

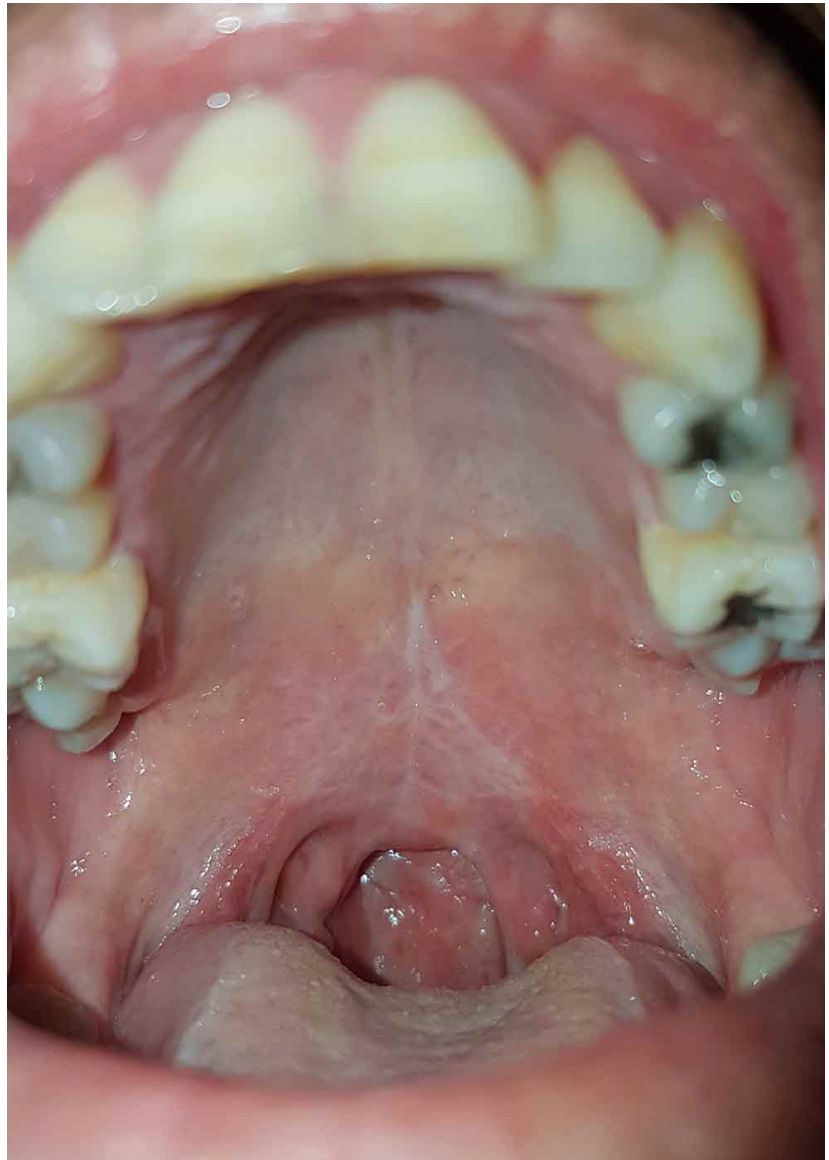

Fig. 5. Control clínico a 30 días de finalizado el tratamiento, evidenciándose notable mejoría.

El paciente fue derivado al Servicio de Dermatología, donde fue medicado con Antimoniato de Meglumina (Glucantime®) $20 \mathrm{mg} / \mathrm{kg} /$ día, por vía intramuscular cada 12 horas, por 30 días. Antes de iniciar el tratamiento se realizó un electrocardiograma, y se evaluó la función hepática, renal y pancreática.

El control clínico se realizó a los 30 días luego de finalizado el tratamiento, evidenciándose una notable mejoría (Fig. 5). Luego se realizaron controles a los 90 y 180 días de finalizado el tratamiento, indicándose controles cada 6 meses durante 2 años.

\section{DISCUSIÓN}

En la LMC puede haber manifestaciones en áreas de mucosa oral y nasal, pudiendo muchas veces llegar a la destrucción del tabique. En esta forma, es común la aparición de una lesión granulomatosa en paladar blando y duro, formando la "Cruz de Escomel", sin embargo, son subdiagnosticadas debido a la inexperiencia del profesional en cuanto a los conocimientos de conceptos de medicina oral y de patologías tropicales. Debemos recordar que esta enfermedad es endémica en nuestra provincia, y las lesiones orales tienden a confundirse con otro tipo de patologías, tales como Paracoccidioidomicosis, Histoplasmosis, Carcinoma Oral de Células Escamosa, Tuberculosis, o Granulomatosis de Wegner, lepra, lo que hace que la LMC sea un diagnóstico diferencial obligado (Passi et al.; Handler et al., 2015b; García Bustos et al., 2016).

El papel que juega la anamnesis es de gran valor para al diagnóstico presuntivo, debido a la importancia de indagar sobre lesiones extraorales previas (como la úlcera de inoculación, que en muchos casos puede ser auto-resolutiva). Los exámenes serológicos en combinación con un examen clínico completo son fundamentales para el diagnóstico de la enfermedad.

En el presente caso, el paciente acudió a la consulta presentando una cicatriz, que podría no haber sido relacionada con la lesión en paladar y en las fosas nasales. Esto dificulta el diagnóstico para quienes no tengan expertise en este tipo de infecciones, por lo que se debe reforzar la importancia del examen clínico extra oral en búsqueda de la cicatriz primaria.

La LMC corresponde solo el $3 \%$ del as leishmaniasis tegumentarias. Las lesiones en piel pueden cicatrizar sin necesariamente haber tenido tratamiento y luego pueden recidivar muchos años después, con lesiones en piel o mucosa oral y/o nasal (Franchini et al., 2017). 
La mayoría de las lesiones secundarias se manifiestan en la mucosa nasal y luego de un tiempo en la mucosa oral, sin embargo, podrían presentarse como primera manifestación como el caso descrito por Almeida et al., donde además reporta otros casos similares con la misma manifestación primaria en cavidad oral.

En base a lo expuesto, la sugerencia es que la muestra se realice con frotis de boca y fosas nasales, complementando con una biopsia.

Debido a la asociación entre Leishmania y VIH en zonas endémicas, es recomendable que el paciente con presentaciones no habituales sean evaluados para infección por $\mathrm{VIH}$ y que tenga garantizado el seguimiento continuo (Alexandrino-de-Oliveira et al., 2010). El paciente presentado proviene de la ciudad de San Ramón de la Nueva Orán, situada en el norte de la provincia de Salta, la cual es una zona endémica para enfermedades tropicales y encontrándose entre las regiones argentinas con mayores casos reportados de leishmaniasis (García Bustos et al., 2016). Sin embargo, el factor geográfico no debería ser exclusivo para el diagnóstico presuntivo de esta enfermedad, ya que esto estaría variando debido a cambios climáticos, deforestaciones y proceso migratorios.

Las pruebas de laboratorio que ayudan a identificar a los microorganismos, pueden ser directas e indirectas. Una de ellas es la toma de biopsia de la lesión, en donde generalmente, las secciones de tejido teñidas con Giemsa o hematoxilina y eosina, permiten observar inclusiones citoplasmáticas macrofagias que pueden sugerir amastigotes de Leishmania. Sin embargo, en la serie de casos de Sánchez-Romero et al., observaron que la tinción con Giemsa no demostró ser útil para confirmar el diagnóstico en casos con amastigotes escasos o no detectables. Además, este grupo reportó que la inmunohistoquímica (IHC) mostró una alta sensibilidad por tinción positiva en 14 de 17 casos (82,3\%), y demostraron que la reacción en cadena de la polimerasa (PCR) es más sensible que la IHC con 13 de 14 (92,8 \%) positivos (Sánchez-Romero et al.).

En este caso, se realizó el diagnóstico con los medios que se tenían al alcance, ya que no en todos los hospitales se disponen de recursos para realizar estudios de IHQ.

Mariz et al. (2019) reportaron un caso de leishmaniasis cutánea difusa, la cual es producida por el complejo L. mexicana, provocando lesiones múltiples y ricas en amastigotes, lo que generalmente es conclu- yente en la biopsia realizada, con posterior tinción de $\mathrm{HE}, \mathrm{IHQ}$ y además el uso de microscopía electrónica de barrido. Estos métodos de estudio son bastante ilustrativos, sin embargo, se sugieren como material didáctico para la docencia, pero poco aplicable a Hospitales con recursos limitados.

En relación al tratamiento de esta enfermedad, se han descrito distintos protocolos, los cuales puede ser en base a medicamentos de uso tópico, intralesionales, intramusculares, orales, intravenosos, o físicos (como crioterapia) y terapia de calor inducida por radiofrecuencia. La elección va a depender de factores como la especie de Leishmania, el tamaño de las lesiones, el estado sistémico del paciente, entre otros (Handler et al., 2015a; Chakravarty \& Sundar, 2019). El protocolo de tratamiento para la LMC indica la utilización de $20 \mathrm{mg} / \mathrm{kg}$ de antimonio pentavalente por 30 días, una vez al día, por vía intramuscular (Organización Panamericana de la Salud, 2013). Drogas alternativas son anfotericina B liposomal, pentamidina, rifampicina o ketoconazol. El presente caso sigue las indicaciones entregadas por la Organización Panamericana de la Salud, publicada el 2013. En esta guía, el uso de antimonios pentavalentes tiene una fuerte fuerza de recomendación, pero baja calidad de evidencia. A pesar de esto, el tratamiento fue efectivo en este paciente, sin presentar recidivas con un seguimiento hasta los 2 años. Es importante que antes de indicar estos fármacos, se evalúe al paciente sistémicamente (tal como se realizó en el presente caso, porque está contraindicado en presencia de alteraciones en el electrocardiograma. Para pacientes con nefropatías, hepatopatías, cardiopatías o con VIH, se recomienda el uso de anfotericina B liposomal (Organización Panamericana de la Salud).

La presente publicación busca alentar a los odontólogos a enriquecer sus conceptos en medicina oral como también estimular la importancia del trabajo interdisciplinario. Las leishmaniasis son consideradas enfermedades reemergentes y un problema para la salud pública mundial, y en el 2009 el $49^{\circ}$ Consejo Directivo de la Organización Panamericana de Salud aprobó la resolución donde se establece el compromiso de fortalecer las acciones de vigilancia y control de las leishmaniasis en América.

CONCLUSIÓN. El conocimiento de las manifestaciones orales puede llevar al diagnóstico clínico de LMC por parte del odontólogo, para diagnóstico y tratamiento oportuno. Debido a las manifestaciones granulomatosas orales, debe considerase a la LMC en el diag- 
nóstico diferencial de muchas patologías que asientan en boca, y debido a su dificultad diagnostica, se aconseja combinar pruebas serológicas, moleculares, exámenes directos, la epidemiologia del paciente y una anamnesis exhaustiva.

MOLINA, A. I. J.; PIMENTEL, S. J. M.; GARCÍA, B. M. F.; PIMENTEL, S. J.; MARCO, J. D.; BRUNETTO, G.; CÓRDOBA, M. \& CORDERO, T. K. Mucocutaneous leishmaniasis with oral manifestation: Case report. Int. J. Odontostomat., 14(3):342-347, 2020.

ABSTRACT: Leishmaniasis is a group of vector-borne diseases caused by Leishmania, an intracellular parasite, which occurs preferentially in tropical and subtropical regions. It manifests itself through a wide range of clinical forms such as cutaneous, mucocutaneous, and visceral, depending on the species and the patient's immune response. We present a case of a 35-year-old man who was referred to the Stomatology Unit of the Señor del Milagro Hospital, Salta, Argentina, presenting in the oral cavity lesion, granulomatous, ulcerated, painful on palpation, unique, soft palate with three months of evolution. Serological, parasitological and PCR studies were performed. Lysed ELISAs, PCRs and cultures of lesion materials were positive, confirming diagnosis of mucocutaneous leishmaniasis. The patient was referred to the Dermatology Service where he received treatment with Meglumine Antimony, with favorable clinical response. The knowledge of the oral manifestations can lead to the clinical diagnosis of mucocutaneous leishmaniasis by the dentist, being able to provide timely treatment and at the same time help the patient, avoiding complications of this disease.

KEY WORDS: mucocutaneous leishmaniasis, tegumentary leishmaniasis, spundia.

\section{REFERENCIAS BIBLIOGRÁFICAS}

Alexandrino-de-Oliveira, P.; Santos-Oliveira, J. R.; Dorval, M. E.; Da-Costa, F.; Pereira, G. R.; da Cunha, R. V.; Paniago, A. M. \& Da-Cruz, A. M. HIVIAIDS-associated visceral leishmaniasis in patients from an endemic area in Central-west Brazil. Mem. Inst. Oswaldo Cruz, 105(5):692-7, 2010.

Almeida, T. F.; da Silveira, E. M.; Dos Santos, C. R.; León, J. E. \& Mesquita, A. T. Exclusive primary lesion of oral leishmaniasis with immunohistochemical diagnosis. Head Neck Pathol., 10(4):533-7, 2016.

Chakravarty, J. \& Sundar, S. Current and emerging medications for the treatment of leishmaniasis. Expert Opin. Pharmacother., 20(10):125165, 2019.

Franchini, R. P.; Fernández Gianotti, C. M.; Cardozo, M. S.; Sacchetti, M. C. \& Terzano, F. E. Lehismaniasis cutáneo-mucosa: presentación de un caso con compromiso medio-facial. Rev. FASO, 24(2):58-62, 2017.

García Bustos, M. F.; Barrio, A.; Parodi Ramoneda, C. M.; Beckar, J.; Moreno, S. \& Basombrío, M. A. M. Miltefosina versus antimoniato de meglumina en el tratamiento de la leishmaniasis mucosa. Medicina (Buenos Aires), 74(5):371-7, 2014.

García Bustos, M. F.; González-Prieto, G.; Ramos, F.; Mora, M. C.; Hashiguchi, Y.; Parodi, C.; Basombrío, M. A.; Moreno, S.; Monroig, S.; Beckar, J.; et al. Clinical and epidemiological features of leishmaniasis in northwestern-Argentina through a retrospective analysis of recent cases. Acta Trop., 154:125-32, 2016.
Germano, M. J.; Salomón, M. C.; Neira, G.; Lozano, E.; Mackern-Oberti, J. P. \& Cargnelutti, D. E. Leishmaniasis in the Argentine Republic: Temporal and geographical distribution from 2013 to 2017. Asian Pac. J. Trop. Med., 12(7):300-5, 2019.

Handler, M. Z.; Patel, P. A.; Kapila, R.; Al-Qubati, Y. \& Schwartz, R. A Cutaneous and mucocutaneous leishmaniasis: Clinical perspectives. J. Am. Acad. Dermatol., 73(6):897-908, 2015a.

Handler, M. Z.; Patel, P. A.; Kapila, R.; Al-Qubati, Y. \& Schwartz, R. A. Cutaneous and mucocutaneous leishmaniasis: Differential diagnosis, diagnosis, histopathology, and management. J. Am. Acad. Dermatol., 73(6):911-26, 2015b.

Hoyos, C. L.; Quipildor, M.; Bracamonte, E.; Lauthier, J. J.; Cajal, P.; Uncos, A.; Korenaga, M.; Hashiguchi, Y.; Barroso, P. A. \& Marco, J. D. Simultaneous occurrence of cutaneous and mucocutaneous leishmaniasis caused by different genotypes of Leishmania (Viannia) braziliensis. J. Dermatol., 46(9):e320-2, 2019.

Lockard, R. D.; Wilson, M. E. \& Rodríguez, N. E. Sex-related differences in immune response and symptomatic manifestations to infection with Leishmania species. J. Immunol. Res., 2019:4103819, 2019.

Marco, J. D.; Barroso, P. A.; Mimori, T.; Locatelli, F. M.; Tomatani, A.; Mora, M. C.; Cajal, S. P.; Nasser, J. R.; Parada, L. A.; Taniguchi, T.; et al. Polymorphism-specific PCR enhances the diagnostic performance of American tegumentary leishmaniasis and allows the rapid identification of Leishmania species from Argentina. BMC Infect. Dis., 12:191, 2012.

Mariz, B. A. L. A.; Sánchez-Romero, C.; Alvarado, N. A. P.; Campos, E. M. M.; de Almeida, O. P. \& Martínez-Pedraza, R. Diffuse cutaneous leishmaniasis with oral involvement in a patient of Northern Mexico. Trop. Doctor, 49(4):303-6, 2019.

Montenegro-ldrogo, J. J.; Montañez-Valverde, R. A.; Chian, C. \& BenitesVillafane, C. Leishmaniasis mucocutánea en un paciente con infección por VIH. Rev. Chil. Infectol., 31(5):610-4, 2014.

Motta, A. C.; Lopes, M. A.; Ito, F. A.; Carlos-Bregni, R.; de Almeida, O. P. \& Roselino, A. M. Oral leishmaniasis: a clinicopathological study of 11 cases. Oral Dis., 13(3):335-40, 2007.

Organización Mundial de la Salud (OMS). Control de las Leishmaniasis: Informe de una Reunión del Comité de Expertos de la OMS sobre el Control de las Leishmaniasis. Ginebra, 22 a 26 de marzo de 2010. Ginebra, Organización Mundial de la Salud, 2012. Disponible en: https:// apps.who.int/iris/bitstream/handle/10665/82766/ WHO TRS 949 spa.pdf?sequence=1\&isAllowed=y

Organización Panamericana de la Salud (OPS). Leishmaniasis en las Américas. Recomendaciones para el Tratamiento. Washington D. C., Organización Panamericana de la Salud, 2013. Disponible en: http:// iris.paho.org/xmlui/handle/123456789/7704

Passi, D.; Sharma, S.; Dutta, S. \& Gupta, C. Localised leishmaniasis of oral mucosa: report of an unusual clinicopathological entity. Case Rep. Dent., 2014:753149, 2014

Salomón, O. D.; Quintana, M. G.; Mastrángelo, A. V. \& Fernández, M. S. Leishmaniasis and climate change-case study:Argentina. J. Trop. Med., 2012:601242, 2012.

Sánchez-Romero, C.; Júnior, H. M.; Matta, V. L. R. D.; Freitas, L. M.; Soares, C. M.; Mariano, F. V.; de Almeida, O. P. \& Nascimento de Aquino, S. Immunohistochemical and molecular diagnosis of mucocutaneous and mucosal Leishmaniasis. Int. J. Surg. Pathol., 8(2):138-45, 2020.

Strazzulla, A.; Cocuzza, S.; Pinzone, M. R.; Postorino, M. C.; Cosentino, S.; Serra, A.; Cacopardo, B. \& Nunnari, G. Mucosal leishmaniasis: an underestimated presentation of a neglected disease. BioMed Res. Int., 2013:805108, 2013.

ul Bari, A. Chronology of cutaneous leishmaniasis: An overview of the history of the disease. J. Pak. Assoc. Dermatol., 16(1):24-7, 2006.

Dirección para correspondencia:

Dr. Ignacio Molina Ávila

Unidad de Estomatología

Hospital Señor del Milagro

Salta - ARGENTINA

Recibido : 03-01-2020

Aceptado: $28-02-2020$

Email: i_molinaavila@hotmail.com 